\title{
Dexamethasone reduces ATDC5 chondrocyte cell viability by inducing autophagy
}

\author{
YAN ZHAO $^{1 *}$, YUAN ZUO $^{2 *}$, HONGJUN HUO $^{1}$, YULONG XIAO $^{1}$, XUEJUN YANG $^{1}$ and DAQI XIN ${ }^{1}$ \\ Departments of ${ }^{1}$ Spine Surgery and ${ }^{2}$ Clinical Laboratory, The Second Affiliated Hospital of \\ Inner Mongolia Medical University, Huimin, Hohhot, Inner Mongolia Autonomous Region 010058, P.R. China
}

Received September 18, 2013; Accepted January 16, 2014

DOI: $10.3892 / \mathrm{mmr} .2014 .1915$

\begin{abstract}
Prolonged use of glucocorticoids (GCs) for the treatment of chronic inflammatory and autoimmune diseases commonly exerts various side-effects, including impairment of skeletal development. However, the effect of GCs on chondrocytes, which play a key role in skeletal development, has been rarely reported. In the present study, autophagy was induced in the ATDC5 chondrocyte cell line following treatment with dexamethasone (Dex) at doses of $1-100 \mu \mathrm{M}$, and that this effect can be inhibited by RU486, a GC antagonist. Autophagy induced by the highest Dex dose $(100 \mu \mathrm{M})$ was associated with a reduction in ATDC5 cell viability. We conclude that high doses of GC can reduce ATDC5 chondrocyte cell viability by inducing autophagy.
\end{abstract}

\section{Introduction}

Glucocorticoids (GCs) are widely used in the local or systemic treatment of chronic inflammatory or autoimmune diseases, and of orthopedic disorders. Prolonged treatment with GCs has various side-effects, including water, salt and in general, metabolic disorders, and impairment of skeletal development (1-3). Administration of GCs leads to a reduction in bone mineral density (BMD), decreased generation of osteoblasts and osteocytes and prolonged lifespan of osteoclasts, with fractures occurring in $30-50 \%$ of patients chronically treated with GCs (1). Recent studies have demonstrated that GCs can induce apoptosis and autophagy in osteocytes (4-6). The influence of GCs has been rarely reported on chondrocytes, which play a key role in skeletal development. Sporadic studies have reported GC effects on the apoptosis and differentiation of chondrocytes $(7,8)$. Autophagy is a lysosomal degradation process that is

Correspondence to: Dr Hongjun Huo or Dr Yulong Xiao, Department of Spine Surgery, Second Affiliated Hospital of Inner Mongolia Medical University, 1 Yingfang Road, Huimin, Hohhot, Inner Mongolia Autonomous Region 010058, P.R. China

E-mail: huohj2013@163.com

E-mail: yu_long_xiao@123.com

${ }^{*}$ Contributed equally

Key words: ATDC5 chondrocyte cells, autophagy, viability essential for cell growth, survival, differentiation, development and homeostasis (9). It is closely regulated that helps to maintain a balance between synthesis, as well as degradation and subsequent recycling of cell products. It is currently unknown whether autophagy occurs in chondrocytes following GC treatment.

In this study, we examined autophagy upon GC treatment in the ATDC5 chondrocyte cell line, and further evaluated the role of autophagy on cell viability. Our findings highlight autophagy as an important mechanism induced by GCs and potentially promoting a reduction in chondrocyte cell viability.

\section{Materials and methods}

Reagents and antibodies. Dexamethasone (Dex), RU486, rapamycin, 3-methyladenine (3-MA) and polyclonal antibodies for glyceraldehyde-3-phosphate dehydrogenase (GAPDH) and microtubule-associated protein 1-light chain 3 (MAP1-LC3) were purchased form Sigma-Aldrich (St. Louis, MO, USA). The polyclonal antibody targeting beclin 1 was purchased from Santa Cruz Biotechnology, Inc. (Santa Cruz, CA, USA). The fusion of the coding sequence of MAP1-LC3 with that of GFP was synthesized and cloned into the pcDNA3.1 ${ }^{+}$vector, to construct the GFP-LC3-expressing plasmid.

Cell culture. The ATDC5 chondrocyte line (Sigma-Aldrich) was cultured in a 1:1 mixture of Dulbecco's modified Eagle's medium (DMEM): Ham's F12, supplemented with $2 \mathrm{mM}$ glutamine and $5 \%$ fetal bovine serum (FBS). We mixed sub-confluent cultures (70-80\%) with trypsin or trypsin/EDTA at a 1:4 ratio; the cells were incubated at $37^{\circ} \mathrm{C}$ in a humidified $5 \% \mathrm{CO}_{2}$ incubator.

Assessment of autophagy with LC3 analysis and electron microscopy. Autophagy was assessed in ATDC5 cells using the GFP-LC3 construct and confocal microscopy analyses. ATDC5 cells grown to $80 \%$ confluency were transfected for $24 \mathrm{~h}$ with the GFP-LC3-expressing plasmid using Lipofectamine 2000 (Invitrogen Life Technologies, Carlsbad, CA, USA). Following transfection, cells were treated with rapamycin (200 nM), Dex $(1,10$ or $100 \mu \mathrm{M})$ or RU486 $(10 \mu \mathrm{M})$ for an additional $24 \mathrm{~h}$ and analyzed by fluorescence microscopy (IX71, Olympus, Tokyo, Japan). In two additional test groups, cells were pretreated with Dex $(10 \mu \mathrm{M})$ and were treated, 2 h later, with 3-MA $(100 \mu \mathrm{M})$ or RU486 $(10 \mu \mathrm{M})$ for $24 \mathrm{~h}$ before examination under a fluorescent microscope. Electron microscopy was performed to determine 

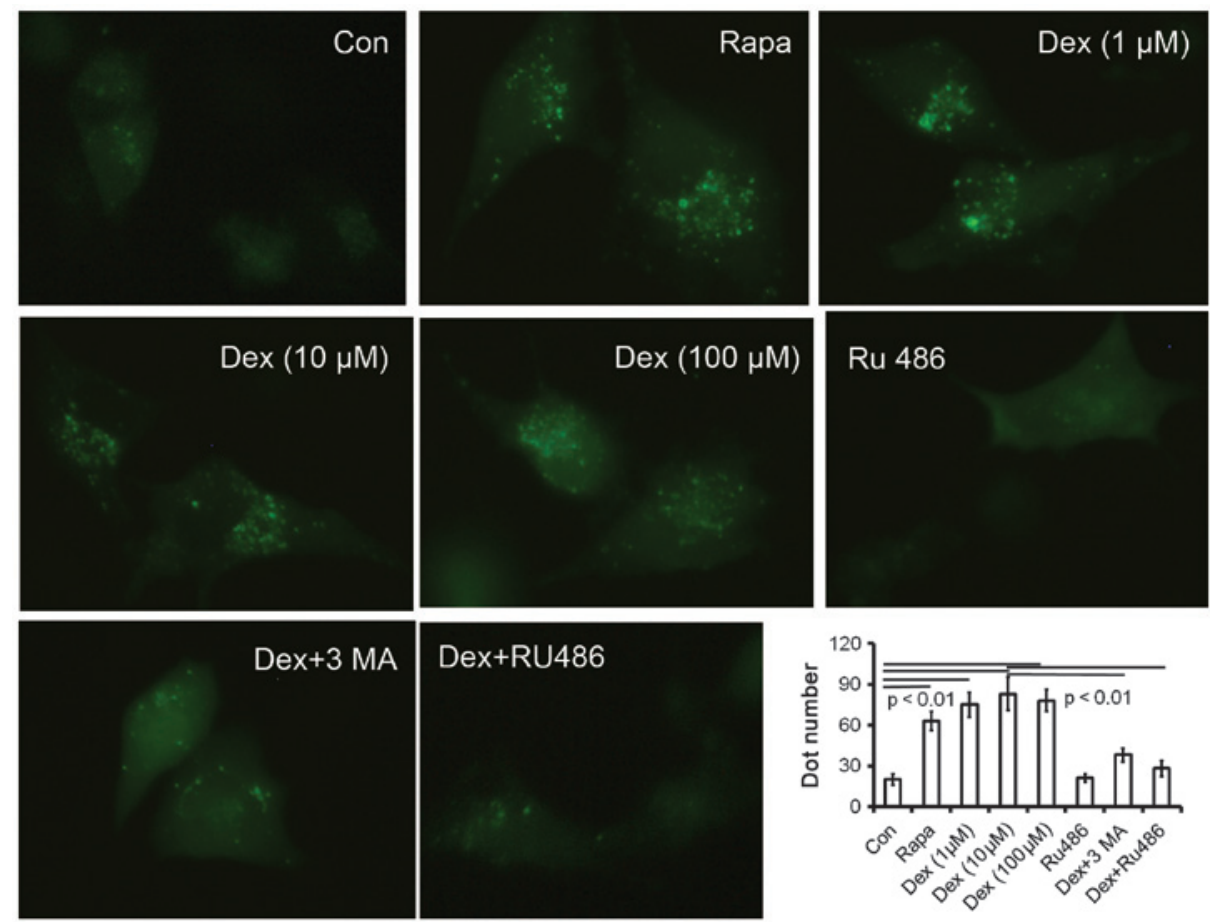

Figure 1. Dexamethasone (Dex) induces autophagic vesicle formation in ATDC5 cells. ADTC5 cells were transfected with a plasmid that expresses a GFP-LC3 fusion protein. After $24 \mathrm{~h}$, cells were incubated for an additional $24 \mathrm{~h}$ at $37^{\circ} \mathrm{C}$ in DMEM: Ham's F12 medium with $1 / 10,000 \mathrm{DMSO}$ (Con), $100 \mathrm{nM}$ rapamycin (Rapa) or Dex $(1,10$ or $100 \mu \mathrm{M})$. Following fixation, the cells were immediately visualized by fluorescence microscopy. The number of punctate GFP-LC3 'dots' in each cell was counted in at least 100 cells/group. The data was normally distributed and was statistically analyzed using the Student-Newman-Keuls test. Horizontal lines denote compared treatments, with $\mathrm{P}<0.01$ considered to indicate significant differences compared to control groups.

the number of autophagic vacuoles in ATDC5 cells treated with or without Dex $(10 \mu \mathrm{M})$. Briefly, ATDC5 cells were washed three times with $1 \mathrm{X}$ phosphate-buffered saline (PBS), trypsinized and collected by centrifugation at $1,000 \mathrm{x} \mathrm{g}$ for $5 \mathrm{~min}$. The cell pellets were fixed in $4 \%$ paraformaldehyde overnight at $4^{\circ} \mathrm{C}$, post-fixed with $1 \% \mathrm{OsO}_{4}$ in cacodylate buffer for $1 \mathrm{~h}$ at room temperature, and gradually dehydrated with ethanol. The dehydrated pellets were rinsed with propylene oxide for $30 \mathrm{~min}$ at room temperature and then embedded in Spurr resin prior to sectioning. Images of thin sections were observed under a transmission electron microscope (JEM 1230; JEOL, Tokyo, Japan).

$R N A$ isolation and reverse transcription-PCR (RT-PCR). For semi-quantitative analysis of the expression levels of mRNAs coding for beclin 1 and Atg 7, total RNA (1-5x10 cells) was extracted with the RNeasy total RNA kit (Qiagen, Hilden, Germany), and reverse transcription was performed on $1 \mu \mathrm{g}$ of RNA/sample using the OneStep RT-PCR kit (Qiagen). Primer sequences and conditions are available upon request. Amplifications were conducted on the resulting cDNAs using a LightCycler instrument (Roche Applied Science, Penzberg, Germany). Data were normalized based on the expression of the $G A P D H$ gene.

Protein isolation and western blot analysis. Whole-cell extracts were prepared by a standard protocol, and proteins were detected by western blot analysis using mouse anti-beclin 1, mouse anti-Atg 7 and rabbit anti-LC3 or GAPDH polyclonal antibodies (Sigma-Aldrich). Anti-mouse or anti-rabbit goat IgG (Pierce Biotechnology, Inc., Rockford, IL, USA) conjugated to horseradish peroxidase were used as the secondary antibody, and the SuperSignal West Femto system (Pierce Biotechnology, Inc., Rockford, IL, USA) was used for enhanced chemiluminescence detection (ECL).

Cell viability assay. Cell viability was determined by the MTT assay. ATDC5 cells were seeded in 96-well plates, and after $24 \mathrm{~h}$, the medium was changed with $\alpha$-minimum essential medium (MEM) containing $1 \%$ FBS, Dex $(0,0.1,1$ or $10 \mu \mathrm{M})$ and/or 3 -MA $(0,50,100$ or $200 \mu \mathrm{M})$. Following incubation for $0,12,24$ or $48 \mathrm{~h}$, the medium was replaced with $50 \mu \mathrm{l}$ of $1 \mathrm{X}$ MTT solution, and the cells were incubated for $2 \mathrm{~h}$ at $37^{\circ} \mathrm{C}$. The MTT solution was then discarded, and $150 \mu \mathrm{l}$ dimethylsulphoxide (DMSO) was added at room temperature to completely dissolve the precipitate. The optical density of the final solution was measured at $570 \mathrm{~nm}$ using a spectrophotometer (Crystaleye; Olympus). Cell viability was expressed as a percentage of viable cells relative to control cells.

Statistical analysis. LC3 dot numbers, expression levels of beclin 1 and Atg 7 and MTT measurements are presented as mean \pm SE. Statistical evaluations of differences in the data were analyzed using the Student's t- or -Newman-Keuls test. $\mathrm{P}<0.05$ was considered to indicate statistical significance.

\section{Results}

Dex induces autophagy and autophagy-associated protein expression in ATDC5 cells. Autophagy is characterized by the formation of numerous acidic vesicles known as acidic vesicular organelles (AVOs) (10). Microphotographs of AVOs were obtained by examining the expression of the GFP-LC3 
A

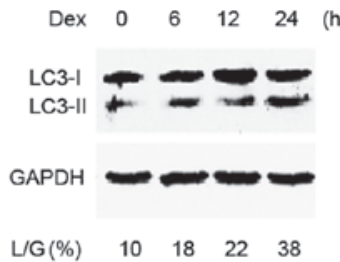

C

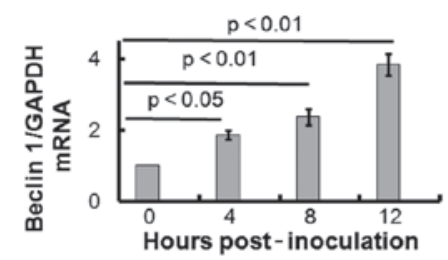

$\mathrm{E}$

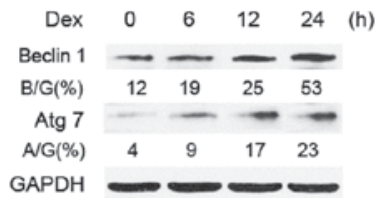

B

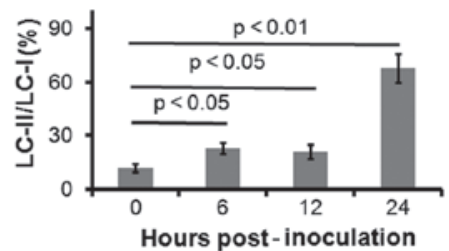

D

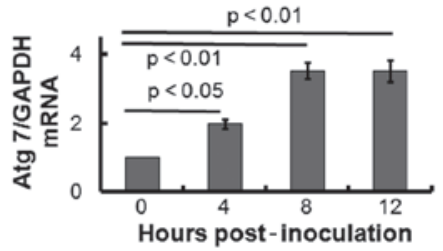

$\mathrm{F}$

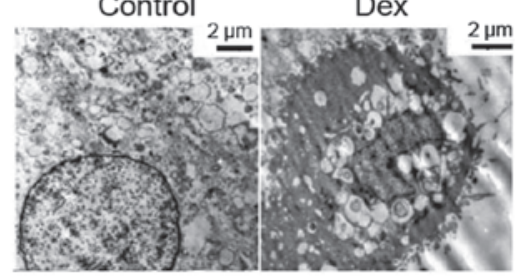

Figure 2. Dexamethasone (Dex) increases the conversion of microtubule-associated protein 1-light chain 3 form I (noted here as LC3-I) to LC3-II and increases beclin 1 and Atg 7 expression at the mRNA and protein levels. (A and B) Following treatment with $10 \mu \mathrm{M}$ Dex for 0-24 h, cells were lysed and subjected to western blot analysis with the indicated antibodies. The ratio of LC3-II) to GAPDH (L/G\%) is presented below the blots. Densitometric analysis was used for quantification. (C and D) mRNA expression of beclin 1 and Atg 7 in ADTC5 cells at $0-12 \mathrm{~h}$ post-treatment with $10 \mu \mathrm{M}$ Dex, analyzed by RT-PCR (E) Western blot analysis of beclin 1 and Atg 7 protein expression levels in ADTC5 cells at 0-12 h post-treatment with $10 \mu \mathrm{M}$ Dex. The ratios of beclin 1 to GAPDH (B/G\%) and Atg7 to GAPDH (A/G\%) are presented below the blots. (F) Electron microscopy of ADTC5 cells following treatment with $10 \mu$ M Dex, as described in Materials and methods. More acidic vesicular organelles were observed in ADTC5 cells compared to control cells.

vector under the fluorescence microscope. Untreated ATDC5 cells showed limited numbers of AVOs in the cytoplasm. Cells treated with rapamycin $(200 \mathrm{nM})$ or Dex $(1,10$ and $100 \mu \mathrm{M})$ demonstrated a significant increase in AVOs (Fig. 1). In addition, it has been reported (11) that the microtubule-associated protein 1-light chain 3 form II (noted here as LC3-II) level increases compared to the LC3-I level during autophagy. To detect the expression of LC3-II, we performed western blot analysis with lysates from ATDC5 cells treated with Dex. The LC3-II/LC3-I expression ratio gradually increased over time in ATDC5 cells treated with Dex (Fig. 2A and B). The autophagy protein beclin 1 is part of a type III phosphatidylinositide (PI) 3 kinase complex, required for the formation of autophagic vesicles (12). In addition, autophagy-related gene (Atg) products, such as Atg 7, play essential roles in autophagy. Fig. 2C-E shows that the expression of beclin 1 and Atg 7 increased postDex treatment, at the mRNA and protein levels. These results indicate that Dex treatment induces autophagy in ATDC5 cells. The ultrastructure of ATDC5 cells treated with or without Dex was examined by electron microscopy (Fig. 2F). Prominent features of autophagy, i.e., cytoplasmic vacuoles and autolysosomes were observed in the cells treated with Dex.

Dex-induced autophagy is inhibited by the GC antagonist RU486 in ATDC5 cells. To further confirm the effect of Dex on autophagy, ATDC5 cells were pretreated with $10 \mu \mathrm{M}$ of the GC antagonist RU486 prior to Dex treatment. Pretreatment with RU486 completely reversed the effect of Dex: microphotographs of the GFP-LC3 reporter vector revealed a decreased number of AVOs in the cells subsequently treated with RU486 or with the autophagy inhibitor 3-MA (Fig. 1), while beclin 1 and Atg 7 mRNA and protein expression levels were significantly decreased (Fig. 3B-D). The results confirmed that Dex can induce autophagy in ATDC5 cells.

ATDC5 cell viability decreases due to Dex-induced autophagy. Dex treatment induced autophagy and increased the expression of autophagy-associated proteins in ATDC5 cells. To further determine the effect of Dex-induced autophagy on cell viability, the MTT assay was conducted in ATDC5 cells. Since Dex was dissolved in $0.5 \%$ ethanol in our experiments, we also evaluated the effect of ethanol on cell viability as a control. Fig. 4A shows that no significant difference in cell viability was observed between the cells treated with or without Dex dissolved in $0.5 \%$ and $1 \%$ ethanol. The ATDC5 cell viability was assayed following treatment with various concentrations of Dex. No significant difference in the viability of ATDC5 cells was detected after treatment with 1 or $10 \mu \mathrm{M}$ Dex for 24-48 h, while the percentage of viable cells following treatment with $100 \mu \mathrm{M}$ Dex was significantly decreased compared to cells not treated with Dex (Fig. 4B and C). Furthermore, to evaluate whether autophagy is involved in the cell viability reduction induced by Dex, the autophagy inhibitor 3-MA was used. Fig. 4D shows that there was no significant difference in ATDC5 cell viability among groups treated with various doses of 3-MA alone; however, the reduction in cell viability induced by $100 \mu \mathrm{M}$ Dex was significantly reverted subsequent to treatment with 100 or $200 \mu \mathrm{M}$ of 3-MA. These results suggest that the viability reduction induced by Dex in ADTC5 cells has been most probably caused by autophagy. 
A

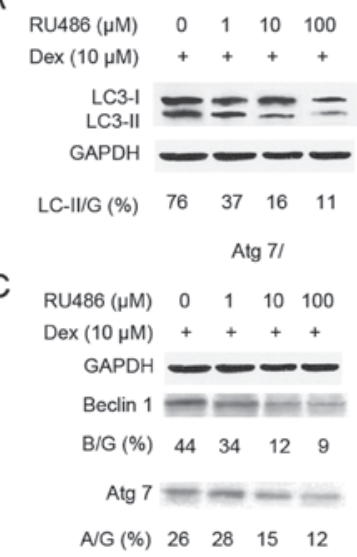

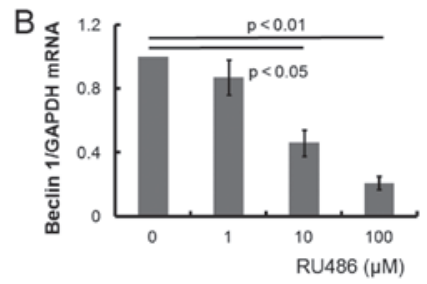

D

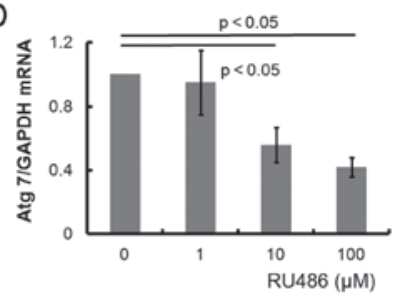

Figure 3. RU486 inhibits expression of the dexamethasone (Dex)-induced autophagy-associated proteins. (A) RU486 inhibited the conversion of microtubule-associated protein 1-light chain 3 form I (noted here as LC3-I) to LC3-II by Dex. Conversion of LC3-I to LC3-II was markedly reduced following treatment with 10 and $100 \mu \mathrm{M}$ RU486. The ratio of LC3-II to GAPDH (L/G\%) is presented below the blots. (B and D) The mRNA level of beclin 1 and Atg 7 was significantly reduced by 10 and $100 \mu \mathrm{M}$ RU486 in ADTC5 cells treated with Dex for $24 \mathrm{~h}$. (C) Beclin 1 and Atg 7 protein expression was significantly reduced by 10 and $100 \mu \mathrm{M}$ RU486 in ADTC5 cells treated with Dex for $24 \mathrm{~h}$.
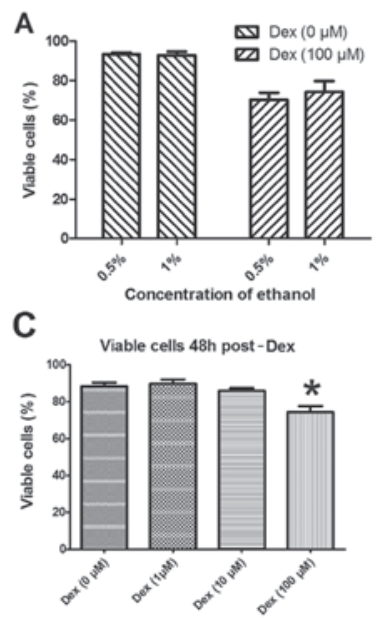
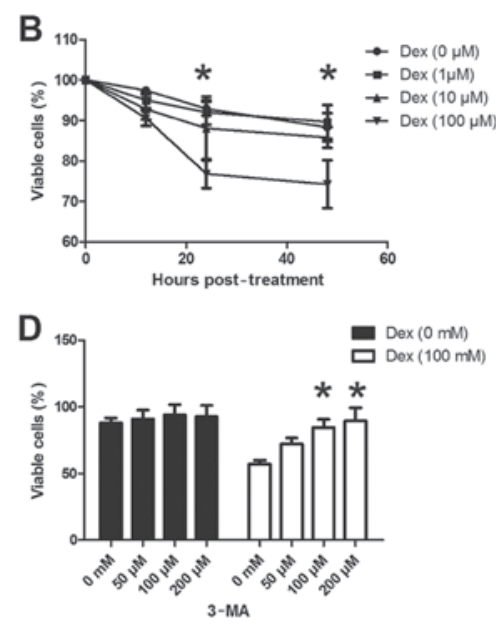

Figure 4. Autophagy induced by a high dose of dexamethasone (Dex) reduces ADTC5 cell viability. (A) Viability of ADTC5 cells treated with Dex dissolved in 0.5 or $1 \%$ ethanol. No significant difference was observed between the cells treated with or without Dex (100 $\mu \mathrm{M})$ dissolved in 0.5 and $1 \%$ ethanol. (B and C) Viability of ADTC5 cells after treatment with $0-100 \mu \mathrm{M}$ Dex. A significant decrease in viability was observed 24 and $48 \mathrm{~h}$ post-inoculation of $100 \mu \mathrm{M}$ Dex, ${ }^{*} \mathrm{P}<0.05$. (D) 3-MA attenuated the Dex-induced ADTC5 cell viability decrease, ${ }^{*} \mathrm{P}<0.05$.

\section{Discussion}

Clinical studies have shown that growth and skeletal development are impaired during treatment with GCs (1-3). In children, maintenance of growth is a complex process that is affected by a number of distinct mechanisms, such as alterations in growth hormone $(\mathrm{GH})$ secretion and in $\mathrm{GH} /$ insulin-like growth factor 1 (IGF-1) sensitivity $(3,13)$. Although compensatory growth often follows the cessation of GC therapy, children with systemic chronic inflammatory diseases subjected to long-term GC treatment may have reduced final height $(14,15)$. Normalizing height in these children, even with concomitant therapy with high-dose recombinant $\mathrm{GH}$, is not straightforward (16-18). Moreover, permanent growth impairment has been reported in children receiving alternate-day GC treatment $(19,20)$. It is well known that the growth plate is responsible for longitudinal bone growth (21). Decreased growth is accompanied by morphological changes in the growth plate in that it becomes thinner $(2,22)$, an effect attributed to decreased proliferation of the chondrocytes $(22,23)$. Detailed studies on this process have revealed that decreased proliferation (22-24) and increased apoptosis $(25,26)$ of epiphyseal chondrocytes contribute to the thinner cartilage.

Autophagy is an intracellular lysosomal (vacuolar) degradation process that is characterized by the formation of double-membrane vesicles in cytoplasm. Since autophagy is involved in cell growth, survival, development and death, the levels of autophagy need to be closely regulated to maintain a balance between synthesis and degradation, and subsequent recycling, of cellular products (27). Autophagy has been proposed to be a 'double-edged sword': it can protect the cells from apoptosis by removing oxidatively damaged organelles, but excess autophagy can destroy cellular components. Autophagy can therefore preserve viability, but also act as a self-destructive process that leads to cell death (28). 
In this study, we detected increased autophagic activity in ATDC5 chondrocytes induced by Dex. Several approaches were adopted to examine autophagy, including counting of fluorescent GFP-LC3 dots, electron microscope imaging and quantification of the relative mRNA and protein expression levels of autophagy-associated molecules. A significant increase in the autophagy-specific AVOs was observed in rapamycin-treated $(200 \mathrm{nM})$ cells or cells treated with 1,10 or $100 \mu \mathrm{M}$ Dex (Fig. 1); the fact that no significant differences in the number of AVOs were found among cells treated with different Dex doses (Fig. 1) may be due to the limits of the used detection method. Alternatively, the time point chosen for examining AVO formation, i.e., $24 \mathrm{~h}$ post-transfection of cells with the GFP-LC3 report vector, may not be the most suitable to discriminate differences among groups. Analyses of the levels of autophagy-associated molecules further confirmed that autophagy is induced by Dex: the LC3-II/LC3-I protein ratio increased in cells post-Dex treatment; beclin 1 and Atg 7 were also more highly expressed in ATDC5 cells post-Dex treatment, at both the both mRNA and protein levels.

To confirm the role of Dex in autophagy in ATDC5 cells, RU486, which is a GC antagonist, was applied to the cells so as to reverse Dex-induced autophagy. As expected, RU486 reduced the Dex-induced autophagy induction (Fig. 1), and the same effect was observed for treatment with the autophagy inhibitor 3-MA. The reversal in autophagy caused by RU486 was confirmed by the reduction in the expression of autophagy-associated molecules (Fig. 3). Taken together, Dex induces autophagy in ATDC5 cells in vitro.

Autophagy was induced in ATDC5 cells by the three doses of Dex tested here, but cell viability was not evenly affected by these doses. Doses of 1 or $10 \mu \mathrm{M}$ Dex appeared to ameliorate, and not reduce, cell viability, although no significant difference was observed compared to the control cells. The 'double-edged sword' feature of the autophagic process was clearly demonstrated by applying the higher dose of Dex: $100 \mu \mathrm{M}$ Dex significantly inhibited ATDC5 cell viability (Fig. 4), and this effect could be reversed by 3-MA. The autophagy induced by the high dose of Dex may thus be sufficient to destroy cellular components, promoting a self-destructive process that eventually leads to cell death. Therefore, autophagy may constitute a novel mechanism involved in the decreased proliferation of chondrocytes following sustained treatment with high GC doses.

In summary, results from this study suggest that GCs can induce autophagy in ATDC5 chondrocytes. In low doses, GCs can exert protective effects, but high doses of GC can induce excess autophagy and reduce cell viability.

\section{Acknowledgements}

This study was supported by grants from funds of the Jilin University Second Hospital and the China-Japan Union Hospital.

\section{References}

1. Canalis E and Delany AM: Mechanisms of glucocorticoid action in bone. Ann N Y Acad Sci 966: 73-81, 2002.

2. Altman A, Hochberg Z and Silbermann M: Interactions between growth hormone and dexamethasone in skeletal growth and bone structure of the young mouse. Calcif Tissue Int 51: 298-304, 1992.
3. Allen DB: Growth suppression by glucocorticoid therapy. Endocrinol Metab Clin North Am 25: 699-717, 1996.

4. Xia X, Kar R, Gluhak-Heinrich J, et al: Glucocorticoid-induced autophagy in osteocytes. J Bone Miner Res 25: 2479-2488, 2010.

5. Weinstein RS, Nicholas RW and Manolagas SC: Apoptosis of osteocytes in glucocorticoid-induced osteonecrosis of the hip. J Clin Endocrinol Metab 85: 2907-2912, 2000.

6. Jia J, Yao W, Guan M, et al: Glucocorticoid dose determines osteocyte cell fate. FASEB J 25: 3366-3376, 2011.

7. Owen HC, Roberts SJ, Ahmed SF and Farquharson C: Dexamethasone-induced expression of the glucocorticoid response gene lipocalin 2 in chondrocytes. Am J Physiol Endocrinol Metab 294: E1023-E1034, 2008.

8. Mushtaq T, Farquharson C, Seawright E and Ahmed SF: Glucocorticoid effects on chondrogenesis, differentiation and apoptosis in the murine ATDC5 chondrocyte cell line. J Endocrinol 175: 705-713, 2002.

9. Mizushima N, Levine B, Cuervo AM and Klionsky DJ: Autophagy fights disease through cellular self-digestion. Nature 451: 1069-1075, 2008.

10. Paglin S, Hollister T, Delohery T, et al: A novel response of cancer cells to radiation involves autophagy and formation of acidic vesicles. Cancer Res 61: 439-444, 2001.

11. Cherra SJ 3rd, Kulich SM, Uechi G, et al: Regulation of the autophagy protein LC3 by phosphorylation. J Cell Biol 190: 533-539, 2010.

12. Sun Y and Peng ZL: Programmed cell death and cancer. Postgrad Med J 85: 134-140, 2009

13. Mushtaq T and Ahmed SF: The impact of corticosteroids on growth and bone health. Arch Dis Child 87: 93-96, 2002.

14. Allen DB, Mullen M and Mullen B: A meta-analysis of the effect of oral and inhaled corticosteroids on growth. J Allergy Clin Immunol 93: 967-976, 1994.

15. Simon D, Lucidarme N, Prieur AM, Ruiz JC and Czernichow P: Treatment of growth failure in juvenile chronic arthritis. Horm Res 58 (Suppl 1): 28-32, 2002.

16. Touati G, Prieur AM, Ruiz JC, Noel M and Czernichow P: Beneficial effects of one-year growth hormone administration to children with juvenile chronic arthritis on chronic steroid therapy. I. Effects on growth velocity and body composition. J Clin Endocrinol Metab 83: 403-409, 1998.

17. Bechtold S, Ripperger P, Muhlbayer D, et al: GH therapy in juvenile chronic arthritis: results of a two-year controlled study on growth and bone. J Clin Endocrinol Metab 86: 5737-5744, 2001.

18. Allen DB, Julius JR, Breen TJ and Attie KM: Treatment of glucocorticoid-induced growth suppression with growth hormone. National Cooperative Growth Study. J Clin Endocrinol Metab 83: 2824-2829, 1998.

19. Sadeghi-Nejad A and Senior B: Adrenal function, growth, and insulin in patients treated with corticoids on alternate days. Pediatrics 43: 277-283, 1969.

20. Lai HC, FitzSimmons SC, Allen DB, et al: Risk of persistent growth impairment after alternate-day prednisone treatment in children with cystic fibrosis. N Engl J Med 342: 851-859, 2000.

21. Baron J, Klein KO, Colli MJ, et al: Catch-up growth after glucocorticoid excess: a mechanism intrinsic to the growth plate. Endocrinology 135: 1367-1371, 1994.

22. Annefeld M: Changes in rat epiphyseal cartilage after treatment with dexamethasone and glycosaminoglycan-peptide complex. Pathol Res Pract 188: 649-652, 1992.

23. Kember NF and Walker KV: Control of bone growth in rats. Nature 229: 428-429, 1971.

24. Mehls O, Himmele R, Homme M, Kiepe D and Klaus G: The interaction of glucocorticoids with the growth hormone-insulin-like growth factor axis and its effects on growth plate chondrocytes and bone cells. J Pediatr Endocrinol Metab 14 (Suppl 6): 1475-1482, 2001.

25. Silvestrini G, Ballanti P, Patacchioli FR, et al: Evaluation of apoptosis and the glucocorticoid receptor in the cartilage growth plate and metaphyseal bone cells of rats after high-dose treatment with corticosterone. Bone 26: 33-42, 2000.

26. Chrysis D, Ritzen EM and Savendahl L: Growth retardation induced by dexamethasone is associated with increased apoptosis of the growth plate chondrocytes. J Endocrinol 176: 331-337, 2003.

27. Neufeld TP: Autophagy and cell growth - the yin and yang of nutrient responses. J Cell Sci 125: 2359-2368, 2012.

28. Tsujimoto Y and Shimizu S: Another way to die: autophagic programmed cell death. Cell Death Differ 12 (Suppl 2): $1528-1534,2005$. 